\title{
La formulación de buenas preguntas en didáctica de la geografía
}

\author{
Jesús Granados Sánchez \\ Universitat de Girona \\ jesus.granados@udg.edu
}

Recibido: julio de 2017

Aceptado: julio de 2017

\section{Resumen}

El conocimiento avanza a medida que se formulan nuevas y buenas preguntas. El presente texto pretende evidenciar la importancia de las preguntas en el avance tanto epistemológico como didáctico de la enseñanza de la geografía. Así, el artículo tiene un doble objetivo: por una parte, se reflexiona sobre la naturaleza de la geografía escolar y se hace una propuesta de cuáles son las grandes cuestiones geográficas de la actualidad. Por otro lado, se considera que plantear buenos interrogantes es una estrategia de gran valor didáctico, puesto que ayuda a alcanzar una mejor comprensión de los fenómenos y crea conciencia. En este aspecto, el texto pretende guiar acerca de cómo formular buenas preguntas, tanto por parte del profesorado como del alumnado, y además aporta una propuesta de diferenciación de tipos de preguntas geográficas según el nivel de demanda intelectual y cognitiva.

Palabras clave: enseñanza de la geografía; grandes preguntas geográficas; buenas preguntas; tipos de preguntas

\section{Resum. La formulació de bones preguntes en didàctica de la geografia}

El coneixement avança a mesura que es formulen noves i bones preguntes. El present text pretén evidenciar la importància de les preguntes en l'avenç tant epistemològic com didàctic de l'ensenyament de la geografia. Així, l'article té un doble objectiu: d'una banda, es reflexiona sobre la disciplina geogràfica escolar i es fa una proposta de les grans qüestions geogràfiques de l'actualitat. D'altra banda, es considera que plantejar bons interrogants constitueix una estratègia de gran valor didàctic, atès que facilita una comprensió més bona dels fenòmens i, a més a més, crea consciència. En aquest aspecte, l'article pretén ajudar a formular bones preguntes geogràfiques, tant per part del professorat com de l'alumnat, i també es fa una proposta de diferenciació de les tipologies de qüestionaments segons el nivell de demanda intel-lectual i cognitiva.

Paraules clau: ensenyament de la geografia; grans qüestions geogràfiques; bones preguntes; tipus de preguntes 
Résumé. Formuler de bonnes questions dans l'enseignement de la géographie

Le savoir progresse au fur et à mesure que de nouvelles et bonnes questions sont soulevées. Cet article vise à montrer l'importance des questions dans le progrès épistémologique et pédagogique de l'enseignement de la géographie. Ainsi, l'article se donne un double objectif: d'une part il réfléchit sur la nature de la géographie scolaire et propose les grandes questions géographiques actuelles; de l'autre il considère que le fait de formuler de bonnes questions est une stratégie d'une grande valeur éducative car elle contribue à une meilleure compréhension des phénomènes et crée une meilleure conscience. Dans cette ligne de pensée, l'article vise à être un guide pour que les enseignants et les élèves puissent formuler de bonnes questions, en même temps qu'il apporte une proposition de différenciation de types de questions selon le niveau intellectuel et cognitif qui sont développés.

Mots-clés: enseignement de la géographie; grandes questions géographiques; bonnes questions; types de questions

\section{Abstract. Good questioning in geography education}

Knowledge advances through the formulation of new and wise questions. The purpose of this paper is to show the importance of questioning and enquiry in the epistemological and pedagogic advancement of geography education. The aim of the paper is twofold: on the one hand, it reflects upon the nature of geography education and proposes a set of big issues or big geographical questions. On the other hand, it considers that the creation of good questions is of great pedagogical value as it helps students to understand complex issues. In this respect, the paper provides a guide for teachers on how to formulate good geographical questions and how to distinguish different levels of cognitive demand in questioning.

Keywords: teaching geography; big geographical questions; good questions; types of questioning; enquiry

\section{Sumario}

1. Introducción 4. ¿¿Qué niveles de preguntas podemos establecer según la demanda cognitiva?

2. ¿Cuáles son las grandes preguntas que pueden guiar la enseñanza de la geografía?

5. Conclusiones

3. ¿Cómo realizar buenas preguntas?

Referencias bibliográficas

\section{Introducción}

En los últimos años, hemos llevado a cabo análisis sobre la tipología de actividades y preguntas que aparecían en los libros de texto de geografía de ESO de Cataluña (Granados Sánchez, 2010 y 2017). Las conclusiones a las que llegan estas investigaciones es que la mayor parte de las actividades de enseñanza y aprendizaje (superior al 80\%) se basan en recordar y comprender, y constituyen una demanda cognitiva baja. Estos resultados nos preocupan y nos han llevado a replantear la formación inicial del profesorado. Desde el curso 2016-2017, 
hemos introducido, en la Universitat de Girona, el trabajo sobre la formulación de preguntas en la enseñanza de la geografía que ayuda a nuestros alumnos a diferenciar que no todas las actividades didácticas planteadas son iguales y que, por tanto, las hay de distintos tipos. Nuestra función ha sido la de aclarar bien y/o diferenciar las tipologías de preguntas, de manera que los profesores en formación inicial puedan establecer buenas actividades de enseñanza y aprendizaje que desarrollen varios estilos de demanda cognitiva.

En este artículo, mostramos el resultado teórico del trabajo que estamos llevando a cabo por medio del debate acerca de la importancia de las preguntas en la formación del pensamiento crítico y como estrategia didáctica en la enseñanza de la geografía. Nuestro propósito es plantear una propuesta que defina las características de las buenas preguntas en la enseñanza de la geografía y establecer un marco que diferencie las cuestiones planteadas según el nivel de demanda cognitiva que suponen, incorporando la metacognición como un nivel superior necesario.

Reinfried y Hertig (2011) afirman que la enseñanza de la geografía desempeña un papel esencial en el desarrollo del pensamiento crítico. Ese tipo de pensamiento está ligado al aprendizaje acerca de cómo investigamos mediante la formulación de preguntas y de cómo las resolvemos. En el caso de la geografía, dichas cuestiones hacen referencia principalmente a las relaciones de la sociedad con el medio ambiente y a las que se establecen entre distintas sociedades en el espacio.

Una pregunta es cualquier oración que tiene una forma o una función interrogativa. En el contexto educativo, las cuestiones que plantea el profesorado se definen como aquellas actividades didácticas o aquellos estímulos que transmiten a los estudiantes para que estos sepan qué y cómo deben aprender (Cotton, 1988). La formulación de preguntas es el método socrático por excelencia. Sirve para comprobar supuestos, encontrar contradicciones y, en definitiva, crear nuevo conocimiento. Formular preguntas es uno de los principales hábitos mentales. Por hábito mental, se entiende la disposición a actuar de forma inteligente ante los problemas, los dilemas y la incertidumbre. Los hábitos mentales definidos por Costa y Kallick (2008) son los siguientes: persistir; manejar la impulsividad; escuchar con atención y empatía; pensar de manera flexible; analizar la propia manera de pensar (metacognición); esforzarse por la exactitud y la precisión; formular preguntas e investigar para la resolución de problemas; aplicar el conocimiento previo a nuevas situaciones; pensar y comunicar con claridad; recopilar información con todos los sentidos; imaginar para crear e innovar; ser curioso y asombrarse por la belleza y la grandeza de la naturaleza y la humanidad; asumir riesgos de forma responsable; encontrar el humor en todas las cosas; trabajar interdependientemente con otros; y tener una actitud abierta al aprendizaje continuo.

El artículo está estructurado en tres apartados principales. En el primero, intentamos reflexionar en torno a la naturaleza de la geografía como disciplina y sobre cuáles son las grandes cuestiones de la geografía escolar. En el segundo apartado, nos centramos en definir qué entendemos por buenas preguntas y 
proponemos cómo deberían formularse. En esta sección, además, indicamos cómo se deben llevar las preguntas al aula después de formularlas para que su aplicación cumpla con su función. A continuación, ponemos énfasis en la importancia de enseñar a los alumnos a plantear sus propias cuestiones y damos orientaciones para guiarlos acerca de cómo mejorar sus respuestas. En el tercer y último apartado, presentamos una propuesta de clasificación de las preguntas en cuatro niveles según la demanda cognitiva y el grado de pensamiento que requieren, ordenados del nivel más simple al superior. La propuesta se justifica a partir de la inclusión de aspectos de distintas taxonomías, como la de Bloom (1956), su revisión llevada a cabo por Anderson y Krathwohl (2001), la taxonomía de Marzano (2001) y la clasificación de Costa (2005).

\section{2. ¿Cuáles son las grandes preguntas que pueden guiar la enseñanza de la geografía?}

La geografía estudia principalmente la localización de las cosas, las condiciones en los lugares y las relaciones y/o las conexiones entre lugares y fenómenos. Así, existen unas razones que explican por qué las cosas están allí donde están. Hay ventajas cuando estas se encuentran en los lugares apropiados y desventajas cuando están en los lugares equivocados. También se puede dar la «ironía geográfica” y las circunstancias que provocan que un lugar sea bueno para ciertas condiciones, a la vez, causen problemas. Algunas de las preguntas que trabaja la geografía son: ¿dónde está? ¿Cómo es? ¿Por qué las cosas se localizan en un sitio determinado? ¿Qué ventajas le propicia? ¿Cuándo pasó y cómo cambia? ¿Qué impacto tiene? ¿Cómo ha de gestionarse de manera que beneficie tanto a la humanidad como al medio ambiente? (Gersmehl, 2008). Para responder a todas estas preguntas, es necesario investigar las localizaciones, los lugares, las situaciones, las regiones, los movimientos, las interacciones entre la humanidad y el medio ambiente, las conexiones y las distribuciones espaciales (IGU-CGE, 2016). Para Huckle (2002), la geografía tiene que explicar cómo funciona el mundo, cómo reconstruimos la naturaleza, cómo nos desarrollamos sosteniblemente y cómo creamos nuestra identidad y nuestro sentido de la responsabilidad como ciudadanos.

La geografía permite realizar distintas miradas sobre el mundo y formar lo que llamamos pensamiento geográfico. Retaillé (2000) describe tres tipos de este: el primero consiste en pensar el mundo como un objeto a ser descrito (es considerado un hábitat cuyas partes tienen un nombre y unas características); la segunda manera consiste en analizar la organización espacial y entender por qué las cosas se localizan allí donde están (el planeta es un marco con unas dimensiones que se miden y se organizan); y la tercera forma de pensamiento consiste en mirar a los actores y a las instituciones del mundo y a su papel en la creación y en la organización del espacio, lo que nos lleva a evaluar su impacto y a valorar las dimensiones culturales, políticas, económicas y simbólicas que influyen en las decisiones (el mundo tiene un significado y una finalidad para cada uno de nosotros). Pero estos tres tipos de pensamiento geográfico no 
desarrollan la agencia o capacidad de acción individual. Por ello, añadimos una cuarta manera de pensar geográficamente, que sería la mirada reflexiva del papel personal en el planeta: cómo mi aprendizaje de la geografía determina la manera en que utilizo el territorio y sus recursos y decido mis acciones de forma consecuente y responsable.

El debate sobre el objeto de estudio de la geografía se ha centrado principalmente en la selección de conceptos clave. De las múltiples listas que se pueden encontrar, hemos seleccionado la propuesta reciente de Butler (2015), que define los siete conceptos clave en geografía siguientes: lugar, espacio, cambio, escala, medio ambiente, sostenibilidad e interconexión. Según el autor, cualquier investigación geográfica ha de centrarse en al menos uno de estos conceptos. Lofthouse (2011) critica esta aproximación de dar relevancia a los grandes conceptos geográficos y prefiere centrarse en las grandes cuestiones o preguntas geográficas. En su opinión, si bien los conceptos geográficos clave pueden ayudar al profesorado a saber qué conocimiento transmitir, estas ideas son difíciles de conceptualizar por parte de los alumnos de la educación obligatoria y no acaban de encontrar sentido a ese aprendizaje. Por ello, propone que resolver las grandes cuestiones geográficas tiene más sentido, puesto que comporta un propósito de aprendizaje en sí mismo (Lofthouse, 2011).

Según la Declaración de Lucerna (Reinfried et al., 2007), para seleccionar las grandes cuestiones geográficas, han de tenerse en cuenta los criterios siguientes:

- Tienen que ser grandes temas del mundo contemporáneo y han de considerar los conflictos que se derivan de la interrelación entre el medio ambiente, la sociedad y la economía.

- Tienen que proporcionar una percepción geográfica del espacio, los lugares y el medio ambiente.

- Se deben utilizar enfoques geográficos para observar la organización espacial.

- Hay que usar ejemplos ilustrativos que sirvan para transferir ideas que se puedan aplicar a otros casos.

- Hay que tener en cuenta las ideas previas, las experiencias y los intereses de los alumnos.

- Las cuestiones deben ser significativas para el alumnado, la sociedad y la ciencia.

- Tiene que haber un equilibrio y una diversidad de temáticas y mostrar diferentes perspectivas que tengan intereses contrarios.

Atendiendo a las aportaciones de Cutter et al. (2002), Lofthouse (2011) y Moude (2009), proponemos las siguientes grandes cuestiones geográficas:

- ¿Qué provoca que los lugares y los paisajes difieran unos de otros y cambien con el tiempo? ¿Por qué eso es importante? 
- ¿Por qué hay una necesidad humana de organizar el espacio a partir de trazar fronteras arbitrarias?

— ¿Qué determina que las personas vivan allí donde lo hacen? ¿Cuándo y por qué las personas, los recursos y las ideas se mueven?

- ¿Por qué existen tantos estilos de vida y tan diversos?

- ¿En qué medida la humanidad ha transformado la Tierra?

- ¿Cómo podemos representar el planeta en que vivimos?

- ¿Cómo podemos aprender sobre el mundo gracias a los avances tecnológicos?

- ¿De qué manera el conocimiento geográfico es importante para la evolución de la civilización humana? ¿Qué papel desempeña en predecir y afrontar el futuro?

- ¿En qué medida la sostenibilidad y la vulnerabilidad cambian de un sitio a otro y a lo largo del tiempo?

- ¿Qué valores sobre el mundo comparten las personas? ¿Cómo afectan los valores y la ideología en las decisiones que se toman y que condicionan nuestras vidas?

\section{3. ¿Cómo realizar buenas preguntas?}

Identificar preguntas y plantear problemas forma parte del proceso de creación de conocimiento, de hacer ciencia. El saber avanza a medida que se formulan nuevas cuestiones y se dan nuevas situaciones. Plantear interrogantes es la columna vertebral de la comunicación entre alumnos y profesores en cualquier proceso de enseñanza y aprendizaje (King, 1999). Una de las tareas más importantes de la práctica docente es el diseño de preguntas (Costa y Kallick, 2015), donde lo importante no es la cantidad (creación de un gran número de ellas), sino su calidad, es decir, hay que formular cuestiones que sean pertinentes para cada objetivo de aprendizaje y apropiadas para el grupo de estudiantes. La pregunta es una aproximación más significativa que crea interés en el alumnado sobre el propio proceso de aprendizaje y facilita la creación de conocimiento y pensamiento (Costa y Kallick, 2000). El profesorado plantea preguntas para averiguar los saberes previos de su alumnado, para motivar e interesar a los jóvenes durante el proceso de aprendizaje, para evaluar los resultados de adquisición de conocimientos, para desarrollar el pensamiento crítico y para hacer conscientes a los alumnos del modo como están aprendiendo.

Siguiendo las contribuciones del AGTA-Geospace (2013), el DfES (2004), Márquez y Roca (2008), Marsden (1995) y Granados Sánchez y Collazo (2017), podemos decir que las buenas preguntas se caracterizan por los siguientes atributos:

- Deben tener un objetivo concreto y la demanda que hagan tiene que ser clara y precisa. Así, el alumnado sabrá qué debe hacer y contestar y su respuesta tendrá relación con lo que nos planteamos. 
- Tienen que mostrar un grado elevado de apertura para desarrollar el pensamiento creativo. Hay que evitar las preguntas cerradas de respuesta corta (sil no, verdaderolfalso, una sola palabra o un único dato).

- Han de estar contextualizadas.

- Hay que tener claros los conocimientos y los conceptos implicados.

- Las preguntas tienen que resultar interesantes al alumnado, así participará activamente en la actividad y propondrá respuestas.

- Las preguntas deben estar en consonancia con el perfil cognitivo del alumnado al cual se dirigen.

- Se deben desarrollar secuencias de cuestiones con diferentes niveles intelectuales y cognitivos que constituyan un reto para los alumnos. Ello comporta conocer taxonomías de preguntas propias de la disciplina.

Una vez generadas las buenas preguntas en base a las características descritas, es importante tener en cuenta una serie de recomendaciones para asegurarnos de que estas cumplen su función: antes que nada, hay que crear un buen clima de aula donde el alumnado se sienta seguro y donde cometer errores se acepte con normalidad; la manera como se pregunte y el tono que se utilice debe ser seductor; se tiene que dar tiempo al alumnado para que pueda responder a las cuestiones; hay que ayudarlo con aclaraciones y sugerencias cuando sea necesario; hay que escuchar activamente sus respuestas y hacer observaciones, siempre reforzando la parte correcta y planteando nuevas interrogaciones sobre aquellos aspectos erróneos.

Habitualmente, se atribuye al profesorado la acción de preguntar. Sin embargo, si queremos que el alumnado desarrolle un alto nivel de autonomía que le permita desarrollar un pensamiento crítico y habilidades de aprendizaje investigativo, debemos procurar enseñarle como debe formular sus propias cuestiones durante el proceso de aprendizaje (Wood, 2006), puesto que se trata de una estrategia de gran valor didáctico que permite desarrollar distintos niveles de autonomía crítica. Plantear interrogantes crea conciencia y ayuda a alcanzar una mejor comprensión de los fenómenos, porque guía la investigación y la resolución de problemas. A través de las preguntas, se le da al estudiante un rol más activo en el aula; se fomenta la motivación, el diálogo y la participación, lo que abre la posibilidad de debatir diferentes opiniones y perspectivas y desarrollar la toma de decisiones.

El alumno ideal es activo, se automotiva, es creativo, se formula cuestiones complejas y busca las respuestas. Sin embargo, como apuntan Graesser y Person (1994), ni todos los estudiantes tienen este perfil ni los centros escolares propician que el alumnado plantee muchos interrogantes. Además, cuando lo hacen, suelen ser superficiales. Catling et al. (2013) sugieren que, para dar la vuelta a esta situación, el profesorado puede actuar de tres maneras: haciendo posible que se investigue a partir de preguntas, mejorando la formulación de cuestiones y empoderando al alumnado. El profesorado tiene que transmitir el sentido de la exploración y la necesidad de investigar aspectos geográficos, y para ello debe permitir que el alumnado plantee sus propias preguntas y lleve a 
cabo su propia investigación. El profesorado puede mejorar la formulación de los interrogantes centrando al alumnado en aquellas cuestiones que son de gran relevancia geográfica y puede empoderarlo dejando que estructure su forma de trabajar y ayudándolo a elegir los métodos de investigación más apropiados.

Por otra parte, los estudiantes pueden encontrar y producir mejores respuestas a las cuestiones que se le plantean si muestra una buena actitud investigadora, si lee bien y comprende la pregunta (¿qué pide?), si crea una respuesta mental inicial donde dibuja qué fuentes de información necesita (¡cómo lo puedo saber?), si es consciente de las limitaciones y de los problemas existentes para responder a esa pregunta y si genera una respuesta amplia que también pueda conducir a nuevos interrogantes (¿qué otras cosas necesito saber?).

\section{4. ¿Qué niveles de preguntas podemos establecer según la demanda cognitiva?}

No todas las preguntas requieren la misma demanda cognitiva. Una que pide al alumno que repita un conocimiento factual es un proceso cognitivo simple, mientras que una que requiere que el estudiante piense de una forma más elaborada y compleja se considera un proceso cognitivo de orden superior. Existen múltiples taxonomías (Bloom, 1956; Gallagher y Aschner, 1963; Marzano y Kendall, 2007) que pretenden guiar al profesorado acerca de cómo desarrollar actividades de enseñanza y aprendizaje de distintos tipos de demanda cognitiva, que conduzcan, a la vez, a la creación de un pensamiento de distinto orden, del más simple al más superior. Para nosotros no hay una única taxonomía que proponga una clasificación de preguntas en niveles cognitivos que sea acorde a nuestra visión de la enseñanza y el aprendizaje de la geografía necesaria en el siglo XXI. La taxonomía de Bloom (1956), pese a tener más de sesenta años, sigue siendo el marco más utilizado por el profesorado. Costa (2005) ha agrupado las seis categorías de la taxonomía de Bloom en tres niveles de preguntas según la complejidad intelectual, y nos parece interesante tener en cuenta dicha simplificación por su sencillez y porque también se centra en otro aspecto nuevo: la importancia de las fuentes de información y cómo las cruzamos y utilizamos para generar nuestro propio conocimiento. En nuestra propuesta, presentamos cuatro niveles de preguntas: mantenemos los tres grados descritos por Costa (2005), que incluyen la recolección de información, el procesamiento de información y la aplicación de la información, y añadimos un cuarto nivel dedicado a la metacognición. A diferencia de Costa (2005), los tres primeros niveles de nuestra propuesta no incluyen la taxonomía original de Bloom, sino la revisión llevada a cabo por Anderson y Krathworlh (2001) y resumida por Krathworlh (2002), que considera, entre otros aspectos, la creación como un proceso cognitivo superior al de evaluación. Aun así, esta revisión no nos sirve para caracterizar la metacognición, ya que la considera como un tipo de conocimiento y no como un proceso cognitivo. Por ello, hemos recurrido a parte de la taxonomía de Marzano (Marzano y Kendall, 2007) para dar forma a nuestro cuarto nivel. Si bien Marzano distingue entre 
el autosistema (self system) como nivel superior y lo separa del sistema metacognitivo, que lo considera como el segundo grado en importancia, nosotros creemos que ambos forman parte de un mismo dominio o nivel.

Para ilustrar nuestra propuesta, partiremos de la metáfora del edificio que presenta Costa (2005). Según él, nuestro pensamiento es como un edificio de tres pisos: el sótano, la planta principal y el ático. En el sótano es donde almacenamos objetos importantes que utilizaremos después. Se trata de información valiosa que aprendemos y guardamos, tales como definiciones, fórmulas, hechos, cifras, etc. En la planta principal es donde entramos a desarrollar el pensamiento, ya que es donde esa información recopilada se utiliza a través de la comparación, el contraste y otras actividades. El ático es como el apartamento de lujo, donde te apropias la información y el conocimiento y te lo haces tuyo. Es donde se sintetiza, se juzga, se evalúa y se crea. Consideramos que esta metáfora es muy potente y útil para entender los diferentes niveles de preguntas. Por ello proponemos completar la metáfora con la inclusión de la metacognición (el nivel 4) a través de una figura humana que mira desde fuera la estructura del edificio y que reflexiona sobre cómo se han construido cada una de las plantas y sobre cuáles son las necesidades y/o reformas que aún precisa el edificio (figura 1).

Figura 1. Los cuatro niveles cognitivos

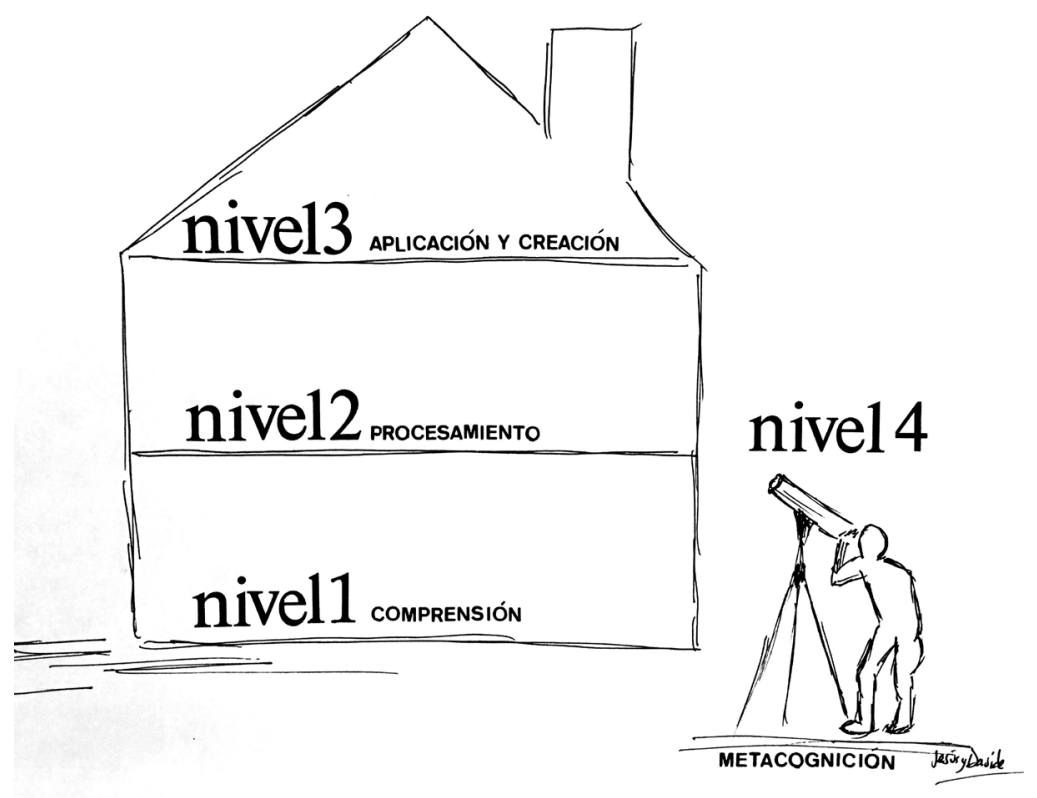

Fuente: elaboración propia a partir de Costa (2005). 
A continuación, se describen los cuatro niveles de preguntas de nuestra propuesta y se distinguen los procesos cognitivos, los verbos indicadores que orientan el tipo de actividad y los ejemplos de cuestiones propias de la enseñanza de la geografía.

Nivel 1. Comprensión y retención de información geográfica. Las preguntas de este nivel pretenden que el alumno recuerde la información o repita procedimientos simples que se hayan trabajado. La pregunta precisa de una respuesta corta que se puede encontrar directamente en las fuentes consultadas (ya sea un texto, un mapa, una fotografía o un vídeo). Se suele solicitar información muy concreta que hace referencia a hechos, datos, términos o conceptos geográficos. Se trata de cuestiones tales como: ¿Quién? ¿Qué? ¿Dónde? ¿Cuándo? También suelen utilizarse preguntas que discriminan entre dos variables (por ejemplo: verdaderolfalso). La respuesta suele consistir en reproducir la información de la misma manera en que aparece en la fuente. Las tareas que hay detrás de este tipo de preguntas implican que el alumnado escuche activamente, sepa recitar de memoria, reconozca los lugares y sus nombres, aprenda nuevos términos geográficos y sepa definir su significado (ver tabla 1).

Nivel 2. Procesamiento de la información geográfica. Las preguntas de este nivel precisan de unas respuestas que normalmente suponen una combinación entre lo aprendido y el conocimiento previo. Normalmente, se han de inferir de la fuente trabajada, es decir, deben ser deducidas. Para ello, el alumnado tiene que analizar en detalle la información para encontrarle sentido y reconocer características y también causas. Así, el pensamiento que se

Tabla 1. Preguntas para la comprensión y la retención de información geográfica (nivel 1)

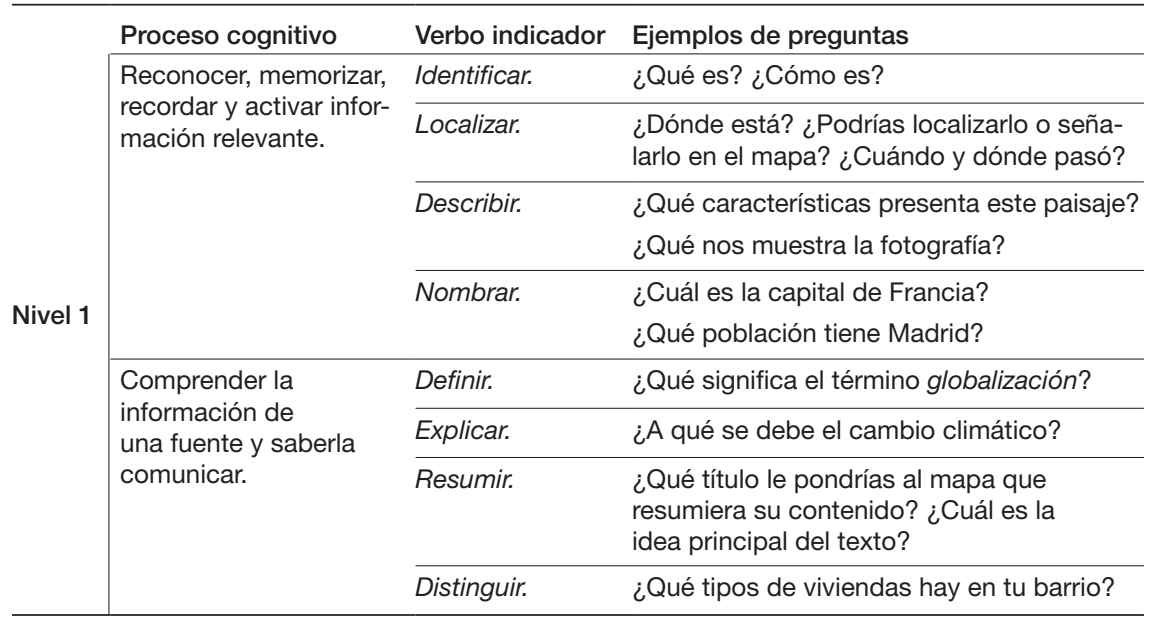

Fuente: elaboración propia. 
Tabla 2. Preguntas para el procesamiento de la información geográfica (nivel 2)

\begin{tabular}{|c|c|c|c|}
\hline & Proceso cognitivo & Verbo indicador & Ejemplos de preguntas \\
\hline & $\begin{array}{l}\text { Aplicar procedimientos } \\
\text { y conocimientos }\end{array}$ & $\begin{array}{l}\text { Aplicar, usar, } \\
\text { implementar. }\end{array}$ & $\begin{array}{l}\text { ¿Qué método utilizarías para resolver este } \\
\text { problema? }\end{array}$ \\
\hline & $\begin{array}{l}\text { adquiridos para } \\
\text { resolver problemas }\end{array}$ & $\begin{array}{l}\text { Representar } \\
\text { gráficamente, }\end{array}$ & $\begin{array}{l}\text { ¿Cómo puedes representar el conflicto de } \\
\text { los refugiados? }\end{array}$ \\
\hline & & ilustrar. & ¿Cómo harías el croquis de tu pueblo? \\
\hline & Analizar problemas a & Diferenciar. & ¿En qué son diferentes? \\
\hline & $\begin{array}{l}\text { partir de descomponer- } \\
\text { los en partes y conocer }\end{array}$ & & $\begin{array}{l}\text { ¿Qué diferencia hay entre las condiciones } \\
\text { de vida en Cuba y España? }\end{array}$ \\
\hline & elementos. & Comparar. & ¿Conoces una situación similar? \\
\hline & & & ¿Qué tienen en común Londres y París? \\
\hline Nivel 2 & & & $\begin{array}{l}\text { ¿En qué se parecen y en qué se diferen- } \\
\text { cian? }\end{array}$ \\
\hline & & Clasificar. & ¿Cómo se clasifican los paisajes? \\
\hline & & & $\begin{array}{l}\text { ¿Qué tipos de estado y de gobierno exis- } \\
\text { ten? }\end{array}$ \\
\hline & & Relacionar. & $\begin{array}{l}\text { ¿Qué relación hay entre las guerras } \\
\text { en Oriente Próximo y los recursos } \\
\text { energéticos? }\end{array}$ \\
\hline & & Deducir. & $\begin{array}{l}\text { ¿Qué valores ideológicos reconoces que } \\
\text { hay detrás de esta propuesta urbanística? }\end{array}$ \\
\hline & & Analizar. & $\begin{array}{l}\text { ¿Qué causa la despoblación de la montaña } \\
\text { y qué consecuencias tiene? }\end{array}$ \\
\hline & & & $\begin{array}{l}\text { ¿Cómo impacta el turismo en la población } \\
\text { de Barcelona? }\end{array}$ \\
\hline
\end{tabular}

Fuente: elaboración propia.

genera implica más de un paso mental e implica comparar, organizar, resumir, secuenciar y analizar. Las respuestas suelen presentar la información de una manera diferente a las presentadas en el libro de texto, las fuentes y/o los recursos utilizados (ver tabla 2).

Nivel 3. Aplicación, creación y evaluación del conocimiento geográfico. Este nivel implica respuestas abstractas que incluyen valoraciones personales que se justifican con evidencias. Para responder a las preguntas, es necesario consultar distintas fuentes, resumir la información, generalizar y transferir conocimiento de un dominio a otro para poder resolver nuevos problemas y aplicar lo que los estudiantes han sintetizado en nuevas situaciones. Las tareas detrás de este tipo de planteamientos incluyen el debate, la predicción, la planificación, la creación, la evaluación, la valoración y la justificación. Este tipo de interrogaciones tiene como objetivo presentar temas conflictivos para conocer qué valores subyacen tras ciertos comportamientos políticos y sociales (y, consecuentemente, qué ideologías) e implican un posicionamiento por parte del alumnado a partir de la expresión y de la justificación de sus opiniones (ver tabla 3). 
Tabla 3. Preguntas para la aplicación, la creación y la evaluación del conocimiento geográfico (nivel 3)

\begin{tabular}{|c|c|c|c|}
\hline \multirow{10}{*}{ Nivel 3} & Proceso cognitivo & Verbo indicador & Ejemplos de preguntas \\
\hline & \multirow[t]{4}{*}{ Evaluar. } & Comprobar. & $\begin{array}{l}\text { ¿Qué evidencias lo demuestran? } \\
\text { ¿Qué contradicciones existen? }\end{array}$ \\
\hline & & Valorar. & $\begin{array}{l}\text { ¿Qué valoración haces de los servicios de tu } \\
\text { ciudad? ¿En base a qué criterios? }\end{array}$ \\
\hline & & Justificar. & $\begin{array}{l}\text { ¿Cómo justificas tu opinión? ¿En qué ideas, } \\
\text { datos y pruebas te apoyas? }\end{array}$ \\
\hline & & $\begin{array}{l}\text { Decidir, escoger, } \\
\text { recomendar. }\end{array}$ & $\begin{array}{l}\text { ¿A qué conclusiones has llegado? } \\
\text { ¿Cuál es tu elección? }\end{array}$ \\
\hline & \multirow[t]{5}{*}{ Crear. } & Planificar. & $\begin{array}{l}\text { ¿Qué medidas y acciones son necesarias } \\
\text { para desarrollar el proyecto? ¿Cómo, cuándo } \\
\text { y quién las llevará a cabo? }\end{array}$ \\
\hline & & $\begin{array}{l}\text { Proponer, } \\
\text { mejorar. }\end{array}$ & $\begin{array}{l}\text { ¿Qué alternativas propones para mejorar } \\
\text { la movilidad urbana? }\end{array}$ \\
\hline & & $\begin{array}{l}\text { Desarrollar, } \\
\text { componer, crear. }\end{array}$ & $\begin{array}{l}\text { ¿Qué has producido para mejorar la } \\
\text { sostenibilidad de tu municipio? ¿Cómo } \\
\text { lo has hecho? ¿Qué aporta tu iniciativa? }\end{array}$ \\
\hline & & \multirow[t]{2}{*}{$\begin{array}{l}\text { Predecir, estimar, } \\
\text { imaginar. }\end{array}$} & $\begin{array}{l}\text { ¿Qué escenarios de futuro pueden derivarse } \\
\text { de los efectos del cambio climático? }\end{array}$ \\
\hline & & & $\begin{array}{l}\text { ¿Cómo te imaginas los medios de transporte } \\
\text { del futuro? }\end{array}$ \\
\hline
\end{tabular}

Fuente: elaboración propia.

Tabla 4. Preguntas metacognitivas para la autorreflexión sobre el aprendizaje en geografía (nivel 4)

\begin{tabular}{|c|c|c|c|}
\hline \multirow{9}{*}{ Nivel 4} & Proceso cognitivo & Dominios & Ejemplos de preguntas \\
\hline & \multirow[t]{8}{*}{ Metacognición. } & Objetivos. & $\begin{array}{l}\text { ¿Qué he aprendido sobre la geografía de la } \\
\text { población? ¿Cuál era su finalidad? }\end{array}$ \\
\hline & & $\begin{array}{l}\text { Importancia } \\
\text { y motivación. }\end{array}$ & $\begin{array}{l}\text { ¿Por qué es relevante saber interpretar el } \\
\text { paisaje? ¿Cómo me ha impactado adquirir } \\
\text { ese conocimiento? ¿Miro el paisaje de otra } \\
\text { forma ahora? }\end{array}$ \\
\hline & & $\begin{array}{l}\text { Respuesta } \\
\text { emocional. }\end{array}$ & $\begin{array}{l}\text { ¿Cómo me ha impacto emocionalmente el } \\
\text { estudio socioeconómico de mi barrio? }\end{array}$ \\
\hline & & Procesos. & $\begin{array}{l}\text { ¿Qué mecanismo utilizas para la } \\
\text { memorización de la localización de los } \\
\text { topónimos en un mapa? }\end{array}$ \\
\hline & & & $\begin{array}{l}\text { ¿Cómo se construye un mapa? ¿Cómo sé } \\
\text { que está bien hecho? }\end{array}$ \\
\hline & & Conexión. & $\begin{array}{l}\text { ¿Cómo se conecta lo aprendido con otros } \\
\text { aprendizajes anteriores en geografía? }\end{array}$ \\
\hline & & & ¿Y con otras disciplinas? \\
\hline & & & ¿Qué lagunas has completado? \\
\hline
\end{tabular}

Fuente: elaboración propia. 
Nivel 4. Metacognición. Este último aspecto constituye un nivel superior que persigue la autorreflexión sobre el proceso de aprendizaje. Se divide en cinco dominios: el primero persigue la conciencia sobre la finalidad o el objetivo de aprendizaje; el segundo interroga al propio individuo acerca de su percepción de la relevancia de ese aprendizaje, lo que estará muy relacionado con el grado de motivación que genere; el tercer dominio tiene que ver con el impacto que ha ocasionado el nuevo conocimiento en las propias emociones; el cuarto considera la conciencia y la supervisión de los procesos acerca de cómo aprende el propio individuo, y, por último, se reflexiona sobre cómo lo aprendido se conecta con otros aprendizajes anteriores y qué emerge de esta conexión (ver tabla 4).

\section{Conclusiones}

La enseñanza de la geografía tiene un papel muy importante en la formación y en el desarrollo del pensamiento crítico de las personas. Por una parte, puede contribuir a ello mediante el conocimiento que se puede generar gracias a la investigación de las grandes cuestiones geográficas y, por otra parte, también con una mejor utilización de la pregunta como estrategia didáctica. Para que eso sea posible, los docentes tienen que reconocer el valor didáctico de las preguntas, saber distinguir con facilidad y plantear cuestiones geográficas con distintos niveles de demanda cognitiva. Kiuhara et al. (2009) afirman que el profesorado frecuentemente plantea preguntas de bajo nivel de pensamiento a sus alumnos. Este tipo de cuestiones, denominadas convergentes por Gallagher y Aschner (1963), requieren una respuesta corta que normalmente repite datos o define conceptos. Su utilización puede deberse a la falta de conocimiento o de experiencia en preguntas de orden superior de pensamiento o por el hecho de sentir presión por llevar a cabo todo el temario establecido en el currículo. Otra razón puede ser el hecho de preparar a los estudiantes para exámenes externos que, normalmente, utilizan este tipo de interrogaciones de bajo nivel de pensamiento y que se centran principalmente en la memorización (Smith y Szymanski, 2013). Si nuestro objetivo es que los jóvenes desarrollen un pensamiento crítico, entonces debemos procurar formular preguntas de los cuatro niveles de demanda cognitiva que hemos propuesto de una manera equilibrada, y especialmente incluir actividades didácticas que fomenten la aplicación, la creación y la evaluación del conocimiento geográfico, así como cuestiones de metacognición, porque estos dos últimos niveles permiten que los alumnos profundicen en el conocimiento y creen conexiones entre todo lo que han aprendido. Pero no debemos olvidar que, como afirman Costa y Kallick (2015), todos los niveles son válidos y necesarios. Lo importante es utilizarlos de manera constante, flexible y no necesariamente de forma progresiva, porque, aunque la mayor parte de las taxonomías son lineales y progresivas, el aprendizaje es recursivo, emergente y aprendemos de forma no lineal (Irvine, 2017). 


\section{Referencias bibliográficas}

ACTA-Geospace (2013). Developing questions [en línea]. <http://www.geogspace.edu. au/verve/_resources/3.8.2_2_dev_questions.pdf>.

ANDERSON, L. y KRATHWOHL, D. (eds.) (2001). A taxonomy for learning, teaching, and assessing: A revision of Bloom's taxonomy of educational objectives-Complete edition. Nueva York: Addison Wesley Longman.

BloOm, B.S. (1956). Taxonomy of Educational Objectives: The classification of educational goals. Handbook I. Cognitive domain. Nueva York: Longman.

Butler, J. (2015). Thinking, Inquiring and Literacy Skills in Geography [en línea]. Taller realizado en el AGTA Conference. <http://www.agta.asn.au/conf2015/ presentations/Butler_J.pdf $>$.

Catling, S.; Willing, T. y Butler, J. (2013). Teaching Primary Geography for Australian Schools. Melbourne: Hawker-Brownlow.

Costa, A. (2005). Arthur Costa's levels of questions [en línea]. Springfield Public Schools. <www.sps186.org/downloads/basic/274780/Costa\%20and\%20Blooms.pdf>.

Costa, A. y KallicK, B. (2000). «Using questions to challenge students' intellect». En: Costa, A. y Kallick, B. (eds.). Activating and engaging habits of mind. Alexandria, VA: Association for Supervision and Curriculum Development, 34-45.

- (2008). Learning and leading with habits of mind: 16 characteristics for success. Alexandria, VA: ASCD.

- (2015). «Five strategies for questionning with intention». Educational Leadership, $73(1), 66-69$.

CotTon, K. (1988). Classroom Questioning. North West Regional Educational Laboratory.

CutTer, S.L.; Golledge, R. y Graf, W. (2002). «The Big Questions in Geography». The Professional Geographer, 54 (3), 305-317. <https://doi.org/10.1111/0033-0124.00332>

DfES (Department for Education And Skills) (2004). Pedagogy and Practice: Teaching and Learning in Secondary Schools. Unit 7: Questioning. Norwick, UK: DfES Publications.

GALLAGHER, J. y ASCHNER, M.J. (1963). «A preliminary report on analysis of classroom interaction». Merrill-Palmer Quarterly of Behaviour and Development, 9, 183-194.

Gersmehl, P. (2008). Teaching Geography. 2. a ed. Nueva York: Guilford.

Graesser, A.C. y Person, N.K. (1994). "Question asking during tutoring». American Educational Research Journal [en línea], 31, 104-137. <https://doi.org/10.3102/00028312031001104>

Granados SÁnCHEZ, J. (2010). L'educació per la sostenibilitat a l'ensenyament de la geografia: Un estudi de cas. Universitat Autònoma de Barcelona. Tesis doctoral.

- (2017). «The level of thinking and questionning of learning activities in Spanish geography textbooks». Comunicación presentada en la Eurogeo: Conference 2017 Amsterdam.

Granados SánCheZ, J. y Collazo, L. (en prensa). «La comprensión y distinción de enfoques interdisciplinarios a partir de la formulación de preguntas en educación ambiental para la sostenibilidad». Enseñanza de las Ciencias.

HuCKLE, J. (2002). «Reconstructing nature: Towards a geographycal education for sustainable development». Geography, 87 (1), 64-72.

IGU-CGE (INTERNATIONAL GEOGRAPHICAL UNION-COMMISSION ON GEOGRAPHICAL EDUCATION) (2016). The International Charter on Geographical Education [en línea]. <http://www.igu-cge.org/Charters-pdf/2016/IGU_2016_def.pdf>. 
IRVINE, J. (2017). «A comparison of revised Bloom and Marzano's New Taxonomy of Learning». Research in Higher Education Journal [en línea], 33. <http://www. aabri.com/manuscripts/172608.pdf>.

KING, S. (1999). "Using questions to promote learning». Teaching Geography, 23 (3), 69-72.

KiuHara, S.A.; Graham, S. y HawKen, L.S. (2009). «Teaching writing to high school students: A national survey». Journal of Educational Psychology [en línea], 101 (1), $136-160$. <https://doi.org/10.1037/a0013097>

KRATHWOHL, D.R. (2002). "A Revision of Bloom Taxonomy: An overview». Theory into Practice, 41 (4), 212-218.

LofThouse, R. (2011). «Is this big enough?: Using big geographical questions to develop subject pedagogy». Teaching Geography, 36 (1), 20-21. Geographical Association.

MÁrqueZ, C. y RocA, M. (2008). «Plantear preguntas: Un punto de partida para aprender ciencias». Revista Educación y Pedagogía, XVIII (45), 61-71.

MARSDEN, W. (1995). Geography 11-16: Rekindling good practice. Londres: David Fulton.

Marzano, R. (2001). Designing a new taxonomy of educational objectives. CA, Thousand Oaks: Corwin.

Marzano, R. y Kendall, J. (2007). The new taxonomy of educational objectives. 2. ${ }^{\text {a }}$ ed. CA, Thousand Oaks: Corwin.

Moude, A. (2009). «Re-centring geography: A school-based perspective on the nature of the discipline». Geographical Research [en línea], 46 (4), 368-379. <https://doi.org/10.1111/j.1745-5871.2009.00589.x>

Reinfried, S. y Hertig, P. (2011). «Geographical Education: How human-environment-society processes work». En: UNESCO-EOLSS JOINT COMMITTEE (eds.). Encyclopedia of Life Support Systems (EOLSS) [en línea]. Oxford, UK: UNESCO, Eolss Publishers. <http://www.eolss.net>.

Reinfried, S.; Schleicher, Y. y Rempfler, A. (eds.) (2007). "Geographical Views on Education for Sustainable Development. Proceedings, Lucerne Symposium Switzerland». Geographiedidaktische Forschungen, 42. Nuremberg: Hochschulverband für Geographie und ihre Didaktik.

Retaillé, D. (2000). «Penser le monde». En: LÉVy, J. y Lussault, M. (eds.). Logiques de l'espace et esprit des lieux: Géographies à Cerisy. París: Belin, 273-286.

Smith, V.G. y Szymanski, A. (2013). "Critical thinking: More than test scores». International Journal of Educational Leadership Preparation, 8 (2), 16-25, NCPEA.

WoOD, P. (2006). «Developing enquiry through questionning». Teaching Geography, $31(2), 76-78$. 